\title{
Synthesis of Amino Acids Analogues of Citric Acid Siderophores
}

\author{
Maria J. Milewska*, Andrzej Chimiak, and John B. Neilands ${ }^{+}$ \\ Department of Organic Chemistry, Technical University of Gdańsk, \\ PL-80-952 Gdańsk, Poland \\ + Department of Biochemistry, University of California, Berkeley, California 94720, USA \\ Z. Naturforsch. 46b, 117-122 (1991); received May 23, 1990 \\ Siderophores, Citric Acid Derivatives, $\omega$-Amino Acid Analogues \\ Three amino acid analogues of citric acid siderophores were synthesized. The synthetic \\ strategy involved condensation of 2-tert-butyl-1,3-di(N-hydroxysuccinimidyl) or 2-tert-butyl- \\ 1,3-bis(pentachlorophenyl)citrates with $\mathrm{N}$-ethyl- $\omega$-aminoalkylhydroxamates. The examina- \\ tion of biological properties of the obtained compounds revealed their poor, if any side- \\ rophore activity and thus confirmed the structural requirements previously suggested for side- \\ rophores of this type.
}

\section{Introduction}

Siderophores [1-3], a large family of natural compounds, play an important role in the iron transport into cells of microorganisms. Some of these compounds, namely aerobactin (1a) [4], arthrobactin (1b) [5] and schizokinen (1c) [6-8] are citric acid derivatives. Schizokinen was isolated from Bacillus megatherium [6] and blue-green algae Anabena [7]. This compound stimulates Anabena growth in the case of iron starvation [9]. Aerobactin was found to be excreted by pathogenic E. coli species and was presumed to be a virulence factor; is probably due to better iron acquisition [10]. Total syntheses of schizokinen, aerobactin and arthrobactin have been already described [11-13]. Recently we have presented [13] a new method of the synthesis of homoschizokinen and schizokinen, which excluded the possibility of imide formation.

The highly specific iron transport system works by forming a siderophore-iron complex and by its competition to a suitable protein receptor [14]. So far, only the aerobactin-iron complex has been studied and the ligands configuration around the central ion has been determined as $\Lambda$ [15]. In this complex, iron is surrounded by oxygen atoms of the hydroxyl and carbonyl groups, and by four oxygen atoms of two N-hydroxyamide bonds. Schizokinen and aerobactin differ from each other

\footnotetext{
Abbreviations: $\mathrm{Pyr}=$ pyridine; $\mathrm{DMF}=$ dimethylformamide; TFA = trifluoroacetic acid.

* Reprint requests to Dr. M. J. Milewska.

Verlag der Zeitschrift für Naturforschung, D-7400 Tübingen 0932-0776/91/0100-0117/\$01.00/0
}

in the length of the carbon chain of the "arms" surrounding the iron ion with a defined radius. This fact must influence the stability of the respective complex and, correspondingly, the siderophore activity of the compound. It is assumed that the schizokinen "arms" are much too short.

\section{Results and Discussion}

In the present work we describe the synthesis and biological properties of three siderophore analogs. These compounds were expected to offer the opportunity for studying the effects of varying arm length on siderophore activity. All the new analogs are of "retro" type i.e. the direction of $\mathrm{N}$-hydroxyamide bonds is opposite to that of natural siderophores. As stated earlier, the biological activity of siderophores is not affected by the "retro" change $[16,17]$. On the other hand, amino acid substrates taken for the syntheses are readily available. Moreover, the methyl group of the acetic acid acyl residue, which is not essential for iron complexation, was replaced by the ethyl group of the easily available $\mathrm{N}$-ethylhydroxylamine. We obtained three "retro"-type analogs $(\mathbf{2 a - c})$ with residues of $\beta$-alanine, $\gamma$-aminobutyric acid and $\varepsilon$-aminocaproic acid. The carboxyl group was disregarded, as it occurs only in aerobactin analogs.

The synthesis ran as follows: carbobenzoxy derivatives of $\beta$-alanine, $\gamma$-aminobutyric and $\varepsilon$-aminocaproic acids $(\mathbf{3 a}-\mathbf{c})$ were transformed into the respective $\mathrm{N}$-ethyl-N-hydroxyamides $(4 \mathbf{a}-\mathbf{c})$ in reaction with $\mathrm{N}$-ethylhydroxylamine (Scheme 2). We tested several methods previously applied for amide synthesis, to finally develop a 
Scheme 1.<smiles>[R]C(CCN(O)C(C)=O)CC([R])NC(=O)NCCC(O)(CC(=O)O)C(=O)O</smiles>

$\begin{array}{lll}\text { 1a: } & \text { R: H } & n: 2 \\ \text { 1b: } & \text { R:H } & n: 4 \\ \text { 1c: } & \text { R: COOH } & n: 4\end{array}$

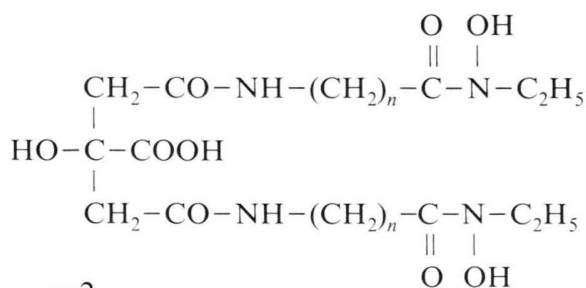

2a: $n: 2$

$\mathrm{O} \mathrm{OH}$

2 b: $n: 3$

2c: $n: 5$

Scheme 2 .

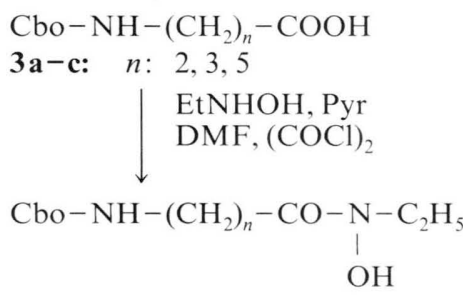

4a-c: $n: 2,3,5$

$$
\mathrm{H}_{2}, 5 \% \mathrm{Pd} / \mathrm{C}
$$
Pyr, EtOH

$\mathrm{NH}_{2}-\left(\mathrm{CH}_{2}\right)_{n}-\mathrm{CO}-\underset{\text { N }}{\mathrm{N}}-\mathrm{C}_{2} \mathrm{H}_{5}$

$\mathbf{5 a - c :} \quad n: 2,3,5$ new method with the use of N,N-dimethylchloromethaniminium chloride [18]. It yielded pure hydroxamic acids $(\mathbf{4} \mathbf{a}-\mathbf{c})$ uncontaminated by $\mathrm{O}$-acyl derivatives. The carbobenzoxy group was then removed from $\mathbf{4 a - c}$ by hydrogenolysis to obtain the respective oily amines $(\mathbf{5} \mathbf{a}-\mathbf{c})$.

5,5-Dimethylenecarboxy-1,3-dioxolan-4-one derivative, which was previously suggested as an useful intermediate in the synthesis of aerobactin [11], finally appeared useless in the synthesis of symmetric diamides of citric acid. Therefore we used DCC and a suitable alcohol to obtain two active esters of citric acid: bis-pentachlorophenyl (7) b) and di-N-hydroxysuccinimidyl (7a) in good yield (Scheme 3 ).

The condensation reaction of the amines $5 \mathbf{a}-\mathbf{c}$ with active esters ( $7 \mathbf{a})$ and $(\mathbf{7} \mathbf{b})$ afforded tert-butyl esters of siderophore analogs $(\mathbf{8} \mathbf{a}-\mathbf{c})$ in good yields, whereafter the tert-butyl group in $\mathbf{8} \mathbf{a}-\mathbf{c}$ was removed with trifluoroacetic acid (Scheme 4). The analogs $\mathbf{2}$ a-c obtained in this way were purified on a Sephadex G-10 column and lyophilized. Their structures were confirmed by mass and NMR spectra for white, hygroscopic lyophilisates. In MS spectra the characteristic fragmentation $[\mathrm{M}-18]^{+}$ was observed. The analog $\mathbf{2}$ a was obtained directly without isolating its tert-butyl ester (8a). The selected synthetic strategy excludes imide formation [13].

Scheme 3 .

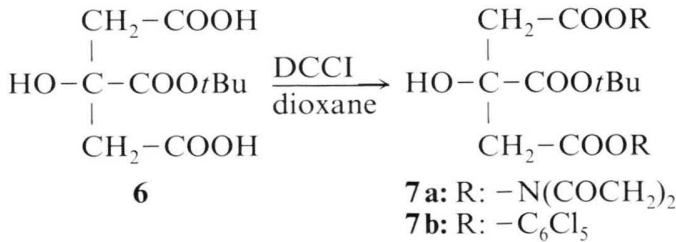

Scheme 4

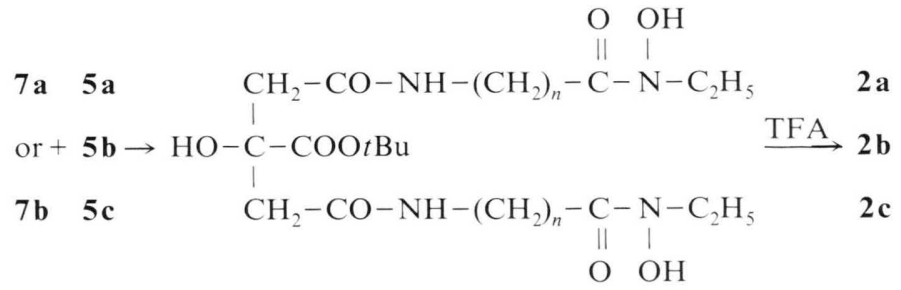

8a: $n: 2$

8b: $n: 3$

8c: $n: 5$ 


\begin{tabular}{lll}
\hline Compound & $\begin{array}{l}\text { E. coli HB 101 } \\
\text { fep A, ent (pABN6) }\end{array}$ & $\begin{array}{l}\text { A.flavescens } \\
\text { ATCC \# 29091 }\end{array}$ \\
\hline $\mathbf{2 a}$ & $\mathrm{NT}$ & - \\
$\mathbf{2 b}$ & + & $\mathrm{NT}$ \\
$\mathbf{2 c}$ & $\mathrm{NT}$ & ++ \\
Rhodotorulic acid & $\mathrm{NT}$ & +++ \\
Aerobactin & ++ & $\mathrm{NT}$ \\
\hline
\end{tabular}

Table I. Structure - siderophore activity of analogues of citric siderophore $\mathbf{2}$.
The data concerning the biological activity of obtained compounds are summarized in Table I.

The siderophore activity of analogues of siderophores (2a) and (2c) were tested in respect $A r$ throbacter flavescens \# ATCC 29091 using rhodotorulic acid as a standard. In this model compound 2 a was found to be inactive, while the activity of $2 \mathbf{c}$ was $2-3$ orders of magnitude lower than that of rhodotorulic acid. On the other hand the analogue $2 \mathbf{b}$ was tested in respect to Escherichia coli $\mathrm{HB} 101$ fep A, ent (pABN6) using aerobactin as a standard. The latter strain is not able to produce or transport enterobactin and harbors a plasmid: pABN 6, which contains only the right extremity of the aerobactin operon. Hence it does not produce any siderophore but can transport compounds of this type and use them for iron supply. The compound $\mathbf{2}$ b showed rather poor siderophore activity in this test (growth zones were $9 \mathrm{~mm}$ for compound $\mathbf{2} \mathbf{b}$ and $18 \mathrm{~mm}$ for aerobactin).

The above data indicate, that as expected, the "arms" of $\mathbf{2} \mathbf{a}$ are too short and therefore this compound cannot act as a siderophore. Compounds $\mathbf{2 b}$ and $\mathbf{2} \mathbf{c}$ contain a bit longer side arms and therefore show some siderophore activity.

Nevertheless both compounds are less active than siderophore of natural orgin.

\section{Experimental}

Melting points are uncorrected. ${ }^{1} \mathrm{H}$ NMR spectra were recorded on Varian EM-360 A, IR spectra on Zeiss-Jena UR-10 apparatus.

\section{Chemicals}

N-Benzyloxycarbonyl- $\beta$-alanine (3a), $\gamma$-amino butyric acid (3b) were prepared acc. [19] (m.p. $106^{\circ} \mathrm{C}$ and $66-67^{\circ} \mathrm{C}$ ). The products have m.p. $102-104{ }^{\circ} \mathrm{C}$ and $65-66^{\circ} \mathrm{C}$, respectively. N-Ben- zyloxycarbonyl- $\varepsilon$-amino-caproic acid (3c) was obtained in a similar way. Yield $68 \%$. The product after crystallization from ethyl acetate/hexane has the m.p. $51-52{ }^{\circ} \mathrm{C}$. - IR $\left(\mathrm{KBr}, \mathrm{cm}^{-1}\right): v_{\mathrm{C}=\mathrm{O}}=$ 1730, 1700. $-{ }^{1} \mathrm{H}$ NMR $\left(\mathrm{CDCl}_{3}, \mathrm{ppm}\right): \delta=1.46$ $\left(\mathrm{m}, 6 \mathrm{H},-\mathrm{CH}_{2}-\right) ; 2.33\left(\mathrm{t}, 2 \mathrm{H},-\mathrm{CH}_{2} \mathrm{CO}\right) ; 3.2(\mathrm{~m}$, $\left.2 \mathrm{H},-\mathrm{C}_{2} \mathrm{NH}^{-}\right) ; 5.1\left(\mathrm{~s}, 2 \mathrm{H},-\mathrm{C}_{2} \mathrm{C}_{6} \mathrm{H}_{5}\right) ; 7.3(\mathrm{~s}$, $\left.5 \mathrm{H},-\mathrm{C}_{6} \mathrm{H}_{5}\right)$.

$$
\begin{array}{rrrr}
\mathrm{C}_{14} \mathrm{H}_{19} \mathrm{O}_{4} \mathrm{~N}(265.3) & & \\
\text { Calcd } & \mathrm{C} 63.34 & \mathrm{H} 7.16 & \text { N } 5.28, \\
\text { Found } & \text { C } 63.61 & \text { H } 6.83 & \text { N 5.26. }
\end{array}
$$

$\mathrm{N}$-Ethyl-N-hydroxyamides of N-benzyloxycarbonyl- $\omega$-amino acids $(\mathbf{4} \mathbf{a}-\mathbf{c})$ were obtained acc. [18].

N-Ethyl-3[(benzyloxycarbonyl)amino]propiohydroxamate (4a) yields $73 \%$; m.p. $64-67{ }^{\circ}$ C; N-ethyl-4[(benzyloxycarbonyl)amino]butyrohydroxamate $(4 \mathbf{b})-57 \%$; m. p. $75-78^{\circ} \mathrm{C}$; N-ethyl-6[(benzyloxycarbonyl)amino]caprohydroxamate (4c) $-80 \%$; m.p. $83-85^{\circ} \mathrm{C}$.

2-tert-Butyl-di-hydrogen citrate (6) and 2-tertbutyl-1,3-di-(N-hydroxysuccinimidyl) citrate (7a) were prepared acc. [13].

\section{$N$-Ethyl-N-hydroxyamides of $\omega$-amino acid $(\mathbf{5} \mathbf{a}-\mathbf{c})$}

N-Ethyl-N-hydroxyamides of N-benzyloxycarbonyl- $\omega$-amino acid (4a), (4b) or $(\mathbf{4 c})(5 \mathrm{mmol})$ was dissolved in $15 \mathrm{ml}$ of ethanol with a few drops of pyridine. The sample was hydrogenated in the presence of $5 \% \mathrm{Pd}$ on charcoal (Koch-Light Lab.) until no Cbo-protected compound existed (TLC test; solvent system chloroform $/ \mathrm{methanol} /$ acetic acid $7: 2: 1$ ). The mixture was filtered, washed with ethanol and water and the solvents evaporated, yielding white, thick oils.

\section{$N$-Ethyl-3-aminopropiohydroxamate (5a)}

Yield 95\%. - ${ }^{1} \mathrm{H}$ NMR $\left(\mathrm{D}_{2} \mathrm{O}, \mathrm{ppm}\right): \delta=1.1(\mathrm{t}$, $\left.3 \mathrm{H}, \mathrm{CH}_{3}-\right) ; 2.3\left(\mathrm{~m}, 2 \mathrm{H},-\mathrm{CH}_{2} \mathrm{CO}\right) ; 3.1(\mathrm{~m}, 4 \mathrm{H}$, $\left.-\mathrm{CH}_{2} \mathrm{~N}\right)$. 
$N$-Ethyl-4-aminobutyrohydroxamate $(\mathbf{5} \mathbf{b})$

Yield 95\% . - ${ }^{1} \mathrm{H}$ NMR (D,O, ppm): $\delta=1.15(\mathrm{t}$, $\left.3 \mathrm{H}, \mathrm{CH}_{3}-\right) ; 1.9\left(\mathrm{~m}, 2 \mathrm{H},-\mathrm{CH}_{2}-\right) ; 2.33(\mathrm{~m}, 2 \mathrm{H}$, $-\mathrm{CH}_{2} \mathrm{CO}$ ); 2.96 (qv, $2 \mathrm{H},-\mathrm{CH}_{2} \mathrm{NH}_{2}$ ); 3.27 (q, $2 \mathrm{H}$, $-\mathrm{CH}_{2} \mathrm{NOH}$ ).

\section{$N$-Ethyl-6-aminocaprohydroxamate $(\mathbf{5 c})$}

Yield 95\%. ${ }^{1} \mathrm{H}$ NMR $\left(\mathrm{D}_{2} \mathrm{O}, \mathrm{ppm}\right): \delta=1.1(\mathrm{t}$, $\left.3 \mathrm{H}, \mathrm{CH}_{3}-\right) ; 1.5\left(\mathrm{~m}, 6 \mathrm{H},-\underline{\mathrm{C}}_{2}-\right) ; 2.45(\mathrm{t}, 2 \mathrm{H}$, $\left.-\mathrm{CH}_{2} \mathrm{CO}\right) ; 3.0\left(\mathrm{~m}, 2 \mathrm{H},-\mathrm{CH}_{2} \mathrm{NH}_{2}\right) ; 3.6(\mathrm{q}, 2 \mathrm{H}$, $-\mathrm{CH}_{2} \mathrm{NOH}$ ).

\section{2-tert-Butyl-1,3-bis (pentachlorophenyl) citrate (7 b)}

To the solution of $2,48 \mathrm{~g}(10 \mathrm{mmol})$ of $(6)$ and $20 \mathrm{mmol}$ of pentachlorophenol in $150 \mathrm{ml}$ dioxane $4,52 \mathrm{~g}(22 \mathrm{mmol})$ of dicyclohexylocarbodiimide in $8 \mathrm{ml}$ ethyl acetate was added dropwise. The reaction was carried out with stirring for $4 \mathrm{~h}$ at room temperature. The urea derivative was filtered off and the solvent evaporated. Then $40 \mathrm{ml}$ of ethyl acetate was added to precipitate the remaining urea derivative. From the filtrate the solvent was evaporated and an active ester recrystallized from $\mathrm{CHCl}_{3} /$ hexane. Yield $6 \mathrm{~g}(80 \%)$; m.p. 178$181{ }^{\circ} \mathrm{C}$. - IR $\left(\mathrm{KBr}, \mathrm{cm}^{-1}\right): v_{\mathrm{C}=\mathrm{O}}=1740,1785$. ${ }^{1} \mathrm{H} \mathrm{NMR}\left(\mathrm{CDCl}_{3}, \mathrm{ppm}\right): \delta=1.5\left(\mathrm{~s}, 9 \mathrm{H}, t-\mathrm{C}_{4} \mathrm{H}_{9}\right)$; $3.3\left(\mathrm{~s}, 4 \mathrm{H},-\mathrm{CH}_{2}-\right)$.

$\begin{array}{rrr}\mathrm{C}_{22} \mathrm{H}_{14} \mathrm{Cl}_{10} \mathrm{O}_{7}(744.90) & \\ \text { Calcd } & \mathrm{C} 35.58 & \mathrm{H} 1.89, \\ \text { Found } & \mathrm{C} 35.74 & \mathrm{H} 1.97 .\end{array}$

tert-Butyl esters of analogues of citric siderophores (8)

A) To a solution of $372 \mathrm{mg}(0,5 \mathrm{mmol}) 2$-tertbutyl-1,3-bis(pentachlorophenyl) citrate (7-b) in $7 \mathrm{ml}$ dioxane $1,18 \mathrm{mmol}$ of $\mathrm{N}$-ethyl-N-hydroxyamide $(\mathbf{5} \mathbf{a}),(\mathbf{5 b})$ or $(\mathbf{5} \mathbf{c})$ were added. Then the solvent was evaporated under reduced pressure and semisolid mixture was extracted several times with ethyl ether to remove a pentachlorophenol. The residue was dissolved in water and passed through a small Zeolite $225\left(\mathrm{H}^{+}, 100-200\right.$ mesh $)$ column. After evaporation, the oily residue was finally purified on silica gel column with benzene-ethyl acetate as solvents.

B) The solution of $221 \mathrm{mg}(0,5 \mathrm{mmol})$ of 2-tertbutyl-1,3-di-N-hydroxysuccinimidyl citrate (7a) in $7 \mathrm{ml}$ of dioxane was treated as in A) with $\mathbf{5} \mathbf{a}, \mathbf{5} \mathbf{b}$ or 5c. Then the solvent removed and the residue was dissolved in $5 \mathrm{ml}$ of water and purified with Zeolite as in A). The filtrate was evaporated to oily residue. The mixture of the product and N-hydroxysuccinimide was finally separated on a silica gel column (eluent as above) or on Sephadex LH-20 column with chloroform-ethyl acetate system (1:1, $\mathrm{v} / \mathrm{v}$ ). Yields and NMR data in the Tables II and III.

\section{Acidolysis of tert-butyl esters}

$0,4 \mathrm{mmol}$ of compounds $\mathbf{8} \mathbf{a}, \mathbf{8} \mathbf{b}$ or $\mathbf{8 c}$ was dissolved in $0,48 \mathrm{ml}(6 \mathrm{mmol})$ of trifluoracetic acid

\begin{tabular}{|c|c|c|c|c|}
\hline Compound & $\begin{array}{l}\text { Procedure } \\
\text { purification }\end{array}$ & $\begin{array}{l}\text { Yield } \\
{[\%]}\end{array}$ & $\begin{array}{l}\text { Mol. formula } \\
\text { (m. weight) }\end{array}$ & $\begin{array}{l}\text { MS-FD } \\
\left(\mathrm{M}^{+}+1\right)\left(\mathrm{M}^{+}\right) \\
\left(\mathrm{M}^{+}-\mathrm{C}_{4} \mathrm{H}_{8}\right)\end{array}$ \\
\hline $8 \mathrm{a}$ & $\begin{array}{l}\text { A } \\
\text { Silica gel }\end{array}$ & 75 & $\begin{array}{l}\mathrm{C}_{20} \mathrm{H}_{36} \mathrm{~N}_{4} \mathrm{O}_{9} \\
(476.5)\end{array}$ & $\begin{array}{l}476(60 \%) \\
420(100 \%)\end{array}$ \\
\hline $8 b$ & $\begin{array}{l}\text { A } \\
\text { Silica gel } \\
\text { B } \\
\text { Sephadex LH } 20\end{array}$ & $\begin{array}{l}50 \\
70\end{array}$ & $\begin{array}{l}\mathrm{C}_{22} \mathrm{H}_{40} \mathrm{~N}_{4} \mathrm{O}_{9} \\
(504.6)\end{array}$ & $\begin{array}{l}504(53 \%) \\
448(100 \%)\end{array}$ \\
\hline $8 \mathrm{c}$ & $\begin{array}{l}\text { A } \\
\text { Silica gel } \\
\text { B } \\
\text { Silica gel } \\
\text { A } \\
\text { Sephadex LH } 20\end{array}$ & $\begin{array}{l}72 \\
74 \\
73\end{array}$ & $\begin{array}{l}\mathrm{C}_{26} \mathrm{H}_{48} \mathrm{~N}_{4} \mathrm{O}_{9} \\
(560.7)\end{array}$ & $\begin{array}{l}560(55 \%) \\
504(100 \%)\end{array}$ \\
\hline $2 a$ & $\begin{array}{l}\text { TFA } \\
\text { Sephadex G } 10\end{array}$ & 94 & $\begin{array}{l}\mathrm{C}_{16} \mathrm{H}_{28} \mathrm{~N}_{4} \mathrm{O}_{9} \\
(420.4)\end{array}$ & $\begin{array}{l}421(40 \%) \\
420(100 \%)\end{array}$ \\
\hline $2 b$ & $\begin{array}{l}\text { TFA } \\
\text { Sephadex G } 10\end{array}$ & 92 & $\begin{array}{l}\mathrm{C}_{18} \mathrm{H}_{32} \mathrm{~N}_{4} \mathrm{O}_{9} \\
(448.5)\end{array}$ & $\begin{array}{l}449(20 \%) \\
448(100 \%)\end{array}$ \\
\hline $2 \mathrm{c}$ & $\begin{array}{l}\text { TFA } \\
\text { Sephadex G } 10\end{array}$ & 92 & $\begin{array}{l}\mathrm{C}_{22} \mathrm{H}_{40} \mathrm{~N}_{4} \mathrm{O}_{9} \\
(504.6)\end{array}$ & $\begin{array}{l}505(23 \%) \\
504(100 \%)\end{array}$ \\
\hline
\end{tabular}

Table II. Physical data of analogues of citric siderophores $\mathbf{2}$ and its tert-butyl esters $\mathbf{8}$. 
Table III. Spectral data of compounds $\mathbf{8}$ and $\mathbf{2}$.

\begin{tabular}{|c|c|c|c|c|c|}
\hline $\begin{array}{l}\text { Com- } \\
\text { pound }\end{array}$ & $\begin{array}{l}\text { IR (film fo } \\
\text { CONOH }\end{array}$ & $\begin{array}{l}\mathrm{r} \mathbf{8}, \mathrm{KBr} \\
\mathrm{CONH}\end{array}$ & $\begin{array}{l}\text { or 2) } v[\mathrm{cn} \\
\mathrm{COOH}\end{array}$ & $\stackrel{-1}{\mathrm{COOO} t \mathrm{Bu}}$ & ${ }^{1} \mathrm{H} \mathrm{NMR}\left(\mathrm{D}_{2} \mathrm{O} / \mathrm{TMS}_{\mathrm{ext}}\right) \delta[\mathrm{ppm}]$ \\
\hline $8 \mathbf{a}$ & 1640 & 1660 & - & 1730 & $\begin{array}{l}1.4\left(\mathrm{~s}, 9 \mathrm{H}, t \mathrm{C}_{4} \mathrm{H}_{9}\right) ; 1.1\left(\mathrm{t}, 6 \mathrm{H}, \mathrm{CH}_{3}\right) ; 2.7\left(\mathrm{~d}, 4 \mathrm{H}, \mathrm{CH}_{2} \mathrm{CO}\right) ; 2.5-2.8 \\
\left(\mathrm{~m}, 4 \mathrm{H}, \mathrm{CH}_{2} \mathrm{CO}\right) ; 3.4\left(\mathrm{t}, 4 \mathrm{H}, \mathrm{CH}_{2} \mathrm{NH}\right) ; 3.6\left(\mathrm{q}, 4 \mathrm{H}, \underline{\mathrm{CH}}_{2} \mathrm{NOH}\right)\end{array}$ \\
\hline $8 b$ & 1640 & 1670 & - & 1730 & $\begin{array}{l}1.4\left(\mathrm{~s}, 9 \mathrm{H}, t \mathrm{C}_{4} \mathrm{H}_{9}\right) ; 1.1\left(\mathrm{t}, 6 \mathrm{H}, \mathrm{CH}_{3}\right) ; 1.8\left(\mathrm{~m}, 4 \mathrm{H}, \mathrm{CH}_{2}\right) ; 2.7(\mathrm{~d}, 4 \mathrm{H}, \\
\left.\mathrm{CH}_{2} \mathrm{CO}\right) ; 2.5\left(\mathrm{~m}, 4 \mathrm{H}, \mathrm{CH}_{2} \mathrm{CO}\right) ; 3.3\left(\mathrm{~m}, 4 \mathrm{H}, \mathrm{CH}_{2} \mathrm{NH}\right) ; 3.6(\mathrm{~m}, 4 \mathrm{H}, \\
\left.\mathrm{C}_{2} \mathrm{NOH}\right)\end{array}$ \\
\hline $8 \mathrm{c}$ & 1635 & 1660 & - & 1730 & $\begin{array}{l}1.4\left(\mathrm{~s}, 9 \mathrm{H}, t \mathrm{C}_{4} \mathrm{H}_{9}\right) ; 1.1\left(\mathrm{t}, 6 \mathrm{H}, \mathrm{CH}_{3}\right) ; 1.5\left(\mathrm{~m}, 12 \mathrm{H}, \mathrm{CH}_{2}\right) ; 2.7(\mathrm{~d}, \\
\left.4 \mathrm{H}, \mathrm{CH}_{2} \mathrm{CO}\right) ; 2.5\left(\mathrm{~m}, 4 \mathrm{H}, \mathrm{CH}_{2} \mathrm{CO}\right) ; 3.1\left(\mathrm{t}, 4 \mathrm{H}, \mathrm{CH}_{2} \mathrm{NH}\right) ; 3.6(\mathrm{q}, \\
\left.4 \mathrm{H}, \mathrm{CH}_{2} \mathrm{NOH}\right)\end{array}$ \\
\hline $2 a$ & 1640 & 1670 & 1715 & - & $\begin{array}{l}1.1\left(\mathrm{t}, 6 \mathrm{H}, \mathrm{CH}_{3}\right) ; 2.7\left(\mathrm{~d}, 4 \mathrm{H}, \mathrm{CH}_{2} \mathrm{CO}\right) ; 2.4-2.9\left(\mathrm{~m}, 4 \mathrm{H}, \mathrm{CH}_{2} \mathrm{CO}\right) \\
3.4\left(\mathrm{t}, 4 \mathrm{H}, \mathrm{CH}_{2} \mathrm{NH}\right) ; 3.6\left(\mathrm{q}, 4 \mathrm{H}, \underline{\mathrm{CH}}_{2} \mathrm{NOH}\right)\end{array}$ \\
\hline $2 b$ & 1640 & 1670 & 1715 & - & $\begin{array}{l}1.1\left(\mathrm{t}, 6 \mathrm{H}, \mathrm{CH}_{3}\right) ; 1.8\left(\mathrm{~m}, 4 \mathrm{H}, \mathrm{CH}_{2}\right) ; 2.7\left(\mathrm{~d}, 4 \mathrm{H}, \mathrm{CH}_{2} \mathrm{CO}\right) ; 2.45(\mathrm{~m}, \\
\left.4 \mathrm{H}, \mathrm{CH}_{2} \mathrm{CO}\right) ; 3.2\left(\mathrm{t}, 4 \mathrm{H}, \mathrm{CH}_{2} \mathrm{NH}\right) ; 3.6\left(\mathrm{q}, 4 \mathrm{H}, \underline{\mathrm{CH}}_{2} \mathrm{NOH}\right)\end{array}$ \\
\hline $2 \mathrm{c}$ & 1635 & 1660 & 1710 & - & $\begin{array}{l}1.1\left(\mathrm{t}, 6 \mathrm{H}, \mathrm{CH}_{3}\right) ; 1.5\left(\mathrm{~m}, 12 \mathrm{H}, \mathrm{CH}_{2}\right) ; 2.4\left(\mathrm{~m}, 4 \mathrm{H}, \mathrm{CH}_{2} \mathrm{CO}\right) ; 2.7(\mathrm{~d}, \\
\left.4 \mathrm{H}, \mathrm{CH}_{2} \mathrm{CO}\right) ; 3.2\left(\mathrm{t}, 4 \mathrm{H}, \mathrm{CH}_{2} \mathrm{NH}\right) ; 3.6\left(\mathrm{q}, 4 \mathrm{H}, \mathrm{CH}_{2} \mathrm{NOH}\right)\end{array}$ \\
\hline
\end{tabular}

and kept at room temperature for 30 minutes. The acid was evaporated and the residue was dissolved in a small amount of water and fractionated on Sephadex G-10 column. The main fraction $\left(\mathrm{FeCl}_{3}\right.$ test) was lyophilized. NMR and MS data of very hygroscopic analogues are in Tables II and III.

\section{Synthesis of $\mathbf{2} \mathbf{a}$ without isolation of tert-butyl ester (8a)}

The equimolar solution of 2-tert-butyl-1,3-di-Nhydroxysuccinimidyl citrate (7a) (1 mmol, $442 \mathrm{mg})$ and $356 \mathrm{mg}(2,1 \mathrm{mmol})$ of N-ethyl3 -aminopropiohydroxamate (5a) hydrochloride in $20 \mathrm{ml}$ of dioxane was treated with $0,3 \mathrm{ml}$ $(2,1 \mathrm{mmol})$ of triethylamine. After $15 \mathrm{~h}$ the solvent was removed and the mixture dissolved in $6 \mathrm{ml}$ of water and passed through Zeolite $225 / \mathrm{H}^{+} /$. The combined fractions containing the desired substance were evaporated and dry residue dissolved in $0,5 \mathrm{ml}$ of trifluoracetic acid. After $30 \mathrm{~min}$ this solution was finally purified using Dowex 1-X4
( $\mathrm{HCOO}^{-}, 100-200$ mesh) column. The crude, glassy, brown solid was dissolved in $40 \mathrm{ml}$ of water mixed with a solution of $150 \mathrm{mg}$ of 8 -oxyquinoline in $40 \mathrm{ml}$ of methanol and stirred for $2 \mathrm{~h}$. Then the water layer was extracted several times with $\mathrm{CHCl}_{3}$ and was lyophilized. Finally this product was on Sephadex G-10 purified as described above and lyophilized once more. The compound is identical with this obtained from tert-butyl ester (8a) (see Tables II and III). Yield $61 \%$.

\section{Biological activity}

The ability of amino acids analogues of citric acid siderophores (2a), (2b), (2c) to support growth of Arthrobacter flavescens \# ATCC 29091 and Escherichia coli $\mathrm{HB} 101 \mathrm{fep} A$, ent (pABN 6) was studied by using the disc diffusion technique on agar surface as described $[17,20]$.

This work was supported by grants CPBR 3.13.6 and CPBP 01.13.2.6. 
[1] J. B. Neilands, Ann. Rev. Biochem. 50, 715 (1981); J. B. Neilands, Ann. Rev. Nutr. 1, 27 (1981); R. J. Bergeron, Chem. Rev. 84, 587 (1984); R. C. Hider, Structure Bonding 58, 25 (1984); A. Chimiak, Postẹpy Biochemii 30, 435 (1984); V. Braun and G. Winkelmann, Prog. Clin. Biochem. Med. 5, 67 (1987).

[2] M. J. Milewska, Z. Chem. 28, 204 (1988).

[3] A. Chimiak and M. J. Milewska, Prog. Chem. Org. Natural Products 53, 203 (1988).

[4] F. Gibson and D. J. Magrath, Biochim. Biophys. Acta 192, 175 (1969)

[5] W. D. Linke, A. Crueger, and H. Diekmann, Arch. Mikrobiol. 85, 44 (1972).

[6] K. B. Mullis, J. R. Pollack, and J. B. Neilands, Biochemistry 10, 4894 (1971)

[7] F. B. Simpson and J. B. Neilands, J. Phycol. 12, 44 (1976).

[8] H. A. Akers, Appl. Environ. Microbiol. 45, 1704 (1983).

[9] T. P. Murphy and D. R. Lean, Science 192, 900 (1976).
[10] P. J. Warner, P. H. Williams, A. Bindereif, and J. B. Neilands, Infect. Immun. 33, 540 (1981); V. Braun, FEMS Microbiol. Lett. 11, 225 (1981).

[11] P. J. Maurer and M. J. Miller, J. Am. Chem. Soc. 104, 3096 (1982).

[12] B. H. Lee and M. J. Miller, J. Org. Chem. 48, 24 (1983).

[13] M. J. Milewska, A. Chimiak, and Z. Głowacki, J. Prakt. Chem. 329, 447 (1987).

[14] J. B. Neilands, Ann. Rev. Microbiol. 36, 285 (1982).

[15] W. R. Harris, C. J. Carrano, and K. N. Raymond, J. Am. Chem. Soc. 101, 2722 (1979).

[16] H. A. Akers, University of California, Ph. D. Thesis, Berkeley (1973).

[17] B. H. Lee, M. J. Miller, C. A. Prody, and J. B. Neilands, J. Med. Chem. 28, 317 (1985).

[18] L. Nakonieczna, M. J. Milewska, T. Kolasa, and A. Chimiak, Synthesis 1985, 929.

[19] R. H. Sifferd and V. du Vigneaud, J. Biol. Chem. 108, 753 (1935); R. L. Evans and F. Irreverre, J. Org. Chem. 24, 863 (1959).

[20] J. B. Neilands, Structure Bonding 58, 1 (1984). 